Pacific

Journal of

Mathematics

\title{
COMPLETE CHARACTERIZATION OF ISOLATED HOMOGENEOUS HYPERSURFACE SINGULARITIES
}

STEPHEN YAU AND HuAiQING ZuO

Volume $273 \quad$ No. 1

January 2015 


\title{
COMPLETE CHARACTERIZATION OF ISOLATED HOMOGENEOUS HYPERSURFACE SINGULARITIES
}

\author{
Stephen Yau AND Huaiqing Zuo
}

\section{Dedicated to Professor Michael Artin on the occasion of his 80th birthday}

Let $X$ be a nonsingular projective variety in $\mathbb{C} \mathbb{P}^{n-1}$. Then the cone over $X$ in $\mathbb{C}^{n}$ is an affine variety $V$ with an isolated singularity at the origin. It is a very natural and important question to ask when an affine variety with an isolated singularity at the origin is a cone over nonsingular projective variety.

This problem is very hard in general. In this paper we shall treat the hypersurface case. Given a function $f$ with an isolated singularity at the origin, we can ask whether $f$ is a weighted homogeneous polynomial or a homogeneous polynomial after a biholomorphic change of coordinates. The former question was answered in a celebrated 1971 paper by Saito. However, the latter question had remained open for 40 years until $\mathrm{Xu}$ and Yau solved it for $f$ with three variables. Recently, Yau and Zuo solved it for $f$ with up to six variables. However, the methods they used are hard to generalize. In this paper, we solve the latter question for general $\boldsymbol{n}$ completely; i.e., we show that $f$ is a homogeneous polynomial after a biholomorphic change of coordinates if and only if $\mu=\tau=(v-1)^{n}$, where $\mu, \tau$ and $v$ are the Milnor number, Tjurina number and multiplicity of the singularity respectively. We also prove that there are at most $\mu^{1 / n}+1$ multiplicities within the same topological type of the isolated hypersurface singularity, while the famous Zariski multiplicity problem asserts that there is only one multiplicity.

\section{Introduction}

Let $w=\left(w_{1}, \ldots, w_{n}\right)$ be an $n$-tuple of positive rational numbers. A polynomial $f\left(z_{1}, \ldots, z_{n}\right)$ is said to be a weighted homogeneous polynomial with weight $w$ if each monomial $\alpha z_{1}^{a_{1}} z_{2}^{a_{2}} \cdots z_{n}^{a_{n}}$ of $f$ satisfies $a_{1} w_{1}+\cdots+a_{n} w_{n}=1$. It has an isolated critical point at $0 \in \mathbb{C}^{n}$ if $\operatorname{grad} f=\left(\partial f / \partial z_{1}, \ldots, \partial f / \partial z_{n}\right)$ is zero at 0 but $\operatorname{grad} f(z) \neq 0$ for all $z$ in a neighborhood of 0 .

Yau was partially supported by the start-up fund from Tsinghua University, and Zuo was partially supported by NSFC no. 11401335 and the start-up fund from Tsinghua University.

MSC2010: primary 32S25; secondary 32S10.

Keywords: homogeneous singularities, Milnor number, multiplicity. 
Recall that a polynomial $f\left(z_{1}, \ldots, z_{n}\right)$ is called quasihomogeneous if $f$ is in the Jacobian ideal of $f$, i.e., $f \in\left(\partial f / \partial z_{1}, \ldots, \partial f / \partial z_{n}\right)$.

By a theorem of Saito (see Theorem 2.7), if $f$ is quasihomogeneous with an isolated critical point at 0 , then after a biholomorphic change of coordinates, $f$ becomes a weighted homogeneous polynomial.

Let $f:\left(\mathbb{C}^{n}, 0\right) \rightarrow(\mathbb{C}, 0)$ be the germ of a complex analytic function with an isolated critical point at the origin. Let $V=\left\{z \in \mathbb{C}^{n}: f(z)=0\right\}$. It is a natural question to ask when $V$ is defined by a weighted homogeneous polynomial up to biholomorphic change of coordinates. Saito [1971] solved this question. He gave a necessary and sufficient condition for $V$ to be defined by a weighted homogeneous polynomial. It is a natural and important question to characterize homogeneous polynomial with an isolated critical point at the origin. This question has remained open for 40 years. In fact it is the first important case of the following interesting problem. Let $X$ be a nonsingular projective variety in $\mathbb{C P ^ { n - 1 }}$. Then the cone over $X$ in $\mathbb{C}^{n}$ is an affine variety $V$ with an isolated singularity at the origin. It is then natural to ask when an affine variety with an isolated singularity at the origin is a cone over nonsingular projective variety.

For a two-dimensional isolated hypersurface singularity $V$, Xu and Yau [1992; 1993] found a coordinate-free characterization when $V$ is defined by a homogeneous polynomial. Recently, necessary and sufficient conditions were given for threedimensional isolated hypersurface singularities with $p_{g} \geq 0$ [Lin and Yau 2004; Lin et al. 2006a; Xu and Yau 1996] and four-dimensional isolated hypersurface singularities with $p_{g}>0$ [Chen et al. 2011], where $p_{g}$ is the geometric genus of the singularity. Based on the classification of weighted homogeneous singularities, Yau and Zuo [2012] solved the problem for $f$ with up to six variables. However, it is quite difficult to generalize their methods to characterize the homogeneous polynomials for general $n$. Ten years ago, Yau formulated the Yau homogeneous characterization conjecture: (1) Let $\mu$ and $v$ be the Milnor number and multiplicity of $(V, 0)$ respectively. Then $\mu \geq(v-1)^{n}$, and equality holds if and only if $f$ is a semihomogeneous function (i.e., $f=f_{v}+g$, where $f_{v}$ is a homogeneous polynomial of degree $v$ defining an isolated singularity at the origin and $g$ consists of terms of degree at least $v+1$ ) after a biholomorphic change of coordinates. (2) Moreover, if $f$ is a weighted homogeneous function, then $\mu=(v-1)^{n}$ if and only if $f$ is a homogeneous polynomial after a biholomorphic change of coordinates. In this paper we verify the Yau homogeneous characterization conjecture affirmatively. As a result, we have solved the characterization problem of homogeneous polynomials with an isolated critical point at the origin, i.e., we have shown that $f$ is a homogeneous polynomial after a biholomorphic change of coordinates if and only if $\mu=\tau=(v-1)^{n}$. 
Recall that the multiplicity of the singularity $V$ is defined to be the order of the lowest nonvanishing term in the power series expansion of $f$ at 0 . The Milnor number $\mu$ and the Tjurina number $\tau$ of the singularity $(V, 0)$ are defined by

$$
\begin{aligned}
\mu & =\operatorname{dim} \mathbb{C}\left\{z_{1}, z_{2}, \ldots, z_{n}\right\} /\left(f_{z_{1}}, \ldots, f_{z_{n}}\right), \\
\tau & =\operatorname{dim} \mathbb{C}\left\{z_{1}, z_{2}, \ldots, z_{n}\right\} /\left(f, f_{z_{1}}, \ldots, f_{z_{n}}\right) .
\end{aligned}
$$

They are numerical invariants of $(V, 0)$.

Let $\pi:(M, A) \rightarrow(V, 0)$ be a resolution of singularity of dimension $n$ with exceptional set $A=\pi^{-1}(0)$. The geometric genus $p_{g}$ of the singularity $(V, 0)$ is the dimension of $H^{n-1}(M, O)$ and is independent of the resolution $M$.

Using $p_{g}, \mu$ and $v$, Yau made another conjecture in 1995 (see [Lin and Yau 2004; Chen et al. 2011]) describing when a weighted homogeneous singularity is a homogeneous singularity. Let $f:\left(\mathbb{C}^{n}, 0\right) \rightarrow(\mathbb{C}, 0)$ be a weighted homogeneous polynomial with an isolated singularity at the origin. Let $\mu, p_{g}$ and $v$ be the Milnor number, geometric genus and multiplicity of the singularity $V=\{z: f(z)=0\}$; then

$$
\mu-p(v) \geq n ! p_{g},
$$

where $p(v)=(v-1)^{n}-v(v-1) \cdots(v-n+1)$, and equality holds if and only if $f$ is a homogeneous polynomial after a biholomorphic change of coordinates.

In fact, we shall prove in this paper that if $p_{g}=0$, then the Yau homogeneous characterization conjecture implies the 1995 Yau conjecture.

These conjectures are sharp estimates and have some important applications in geometry. However, they were proved only for low-dimensional singularities. For the Yau homogeneous characterization conjecture, Lin, Wu, Yau and Luk [Lin et al. 2006b] proved the following two theorems.

Theorem 1.1. Let $f:\left(\mathbb{C}^{2}, 0\right) \rightarrow(\mathbb{C}, 0)$ be the germ of a holomorphic function defining an isolated plane curve singularity $V=\left\{z \in \mathbb{C}^{2}: f(z)=0\right\}$ at the origin. Let $\mu$ and $v$ be the Milnor number and multiplicity of $(V, 0)$, respectively. Then

$$
\mu \geq(v-1)^{2} .
$$

Furthermore, if $V$ has at most two irreducible branches at the origin, or if $f$ is a quasihomogeneous function, then equality holds in (1-1) if and only if $f$ is a homogeneous polynomial (after a biholomorphic change of coordinates).

Theorem 1.2. Let $f:\left(\mathbb{C}^{n}, 0\right) \rightarrow(\mathbb{C}, 0)$ be the germ of a holomorphic function defining an isolated hypersurface singularity $V=\left\{z \in \mathbb{C}^{n}: f(z)=0\right\}$ at the origin. Let $\mu, v$ and $\tau=\operatorname{dim} \mathbb{C}\left\{z_{1}, \ldots, z_{n}\right\} /\left(f, \partial f / \partial z_{1}, \ldots, \partial f / \partial z_{n}\right)$ be the Milnor number, multiplicity and Tjurina number of $(V, 0)$, respectively. Suppose $\mu=\tau$ and $n$ is either 3 or 4 . Then

$$
\mu \geq(v-1)^{n},
$$


and equality holds in (1-2) if and only if $f$ is a homogeneous polynomial (after a biholomorphic change of coordinates).

For the 1995 Yau conjecture, Lin, Tu and Yau [Lin and Yau 2004; Lin et al. 2006a] have the following theorem:

Theorem 1.3. Let $(V, 0)$ be a three-dimensional isolated hypersurface singularity defined by a weighted homogeneous polynomial $f(x, y, z, w)=0$. Let $\mu, v$ and $p_{g}$ be the Milnor number, multiplicity and geometric genus of the singularity, respectively. Then

$$
\mu-\left(2 v^{3}-5 v^{2}+2 v+1\right) \geq 4 ! p_{g}
$$

and equality holds in (1-3) if and only if $f$ is a homogeneous polynomial.

Remark. The above theorem is proved in [Lin and Yau 2004] with $p_{g}>0$. For $p_{g}=0$, the theorem is proved in [Lin et al. 2006a].

An immediate corollary of Theorem 1.3 is the following:

Corollary 1.4 [Lin et al. 2006a]. Let $(V, 0)$ be a three-dimensional isolated hypersurface singularity defined by a polynomial $f(x, y, z, w)=0$. Let $\mu, v, p_{g}$ and $\tau$ be the Milnor number, multiplicity, geometric genus and Tjurina number of the singularity, respectively. Then $f$ is a homogeneous polynomial after a biholomorphic change of coordinates if and only if $\mu=\tau$ and $\mu-\left(2 v^{3}-5 v^{2}+2 v+1\right)=4 ! p_{g}$.

Chen, Lin, Yau and Zuo generalized the above theorem to four-dimensional isolated hypersurface singularities with the additional assumption $p_{g}>0$.

Theorem 1.5 [Chen et al. 2011]. Let $(V, 0)$ be a four-dimensional isolated hypersurface singularity defined by a weighted homogeneous polynomial $f(x, y, z, w, t)=0$. Let $\mu, v$ and $p_{g}$ be the Milnor number, multiplicity and geometric genus of the singularity, respectively. If $p_{g}>0$ then

$$
\mu-\left[(v-1)^{5}+v(v-1)(v-2)(v-3)(v-4)\right] \geq 5 ! p_{g},
$$

and equality holds in (1-4) if and only if $f$ is a homogeneous polynomial after a biholomorphic change of coordinates.

Corollary 1.6 [Chen et al. 2011]. Let $(V, 0)$ be a four-dimensional isolated hypersurface singularity defined by a polynomial $f(x, y, z, w, t)=0$. Let $\mu, v, p_{g}$ and $\tau$ be the Milnor number, multiplicity, geometric genus and Tjurina number of the singularity, respectively. Moreover, if $p_{g}>0$ then $f$ is a homogeneous polynomial after a biholomorphic change of coordinates if and only if $\mu=\tau$ and

$$
\mu-\left[(v-1)^{5}+v(v-1)(v-2)(v-3)(v-4)\right]=5 ! p_{g} .
$$

Yau and Zuo [2012] proved the following theorem: 
Theorem 1.7. Let $f:\left(\mathbb{C}^{k}, 0\right) \rightarrow(\mathbb{C}, 0)$, where $k$ is either 5 or 6 , be a polynomial with an isolated singularity at the origin. Let $\mu, v$ and $\tau$ be the Milnor number, multiplicity and Tjurina number of the singularity $V=\{z: f(z)=0\}$ respectively. Then $f$ is a homogeneous polynomial after a biholomorphic change of coordinates if and only if $\mu=\tau=(\nu-1)^{k}$.

The purpose of this paper is to prove the following results:

Theorem A. Let $f:\left(\mathbb{C}^{n}, 0\right) \rightarrow(\mathbb{C}, 0)$ be a weighted homogeneous polynomial with an isolated singularity at the origin. Let $\mu$ and $v$ be the Milnor number and multiplicity of the singularity $V=\{z: f(z)=0\}$ respectively. Then

$$
\mu \geq(v-1)^{n},
$$

and equality holds in (1-5) if and only if $f$ is a homogeneous polynomial after a biholomorphic change of coordinates.

Corollary B. Let $f:\left(\mathbb{C}^{n}, 0\right) \rightarrow(\mathbb{C}, 0)$ be a polynomial with an isolated singularity at the origin. Let $\mu, v$ and $\tau$ be the Milnor number, multiplicity and Tjurina number of the singularity $V=\{z: f(z)=0\}$ respectively. Then $f$ is a homogeneous polynomial after a biholomorphic change of coordinates if and only if $\mu=\tau=(\nu-1)^{n}$.

Proposition 3.1 and Theorem A answer the Yau homogeneous characterization conjecture affirmatively, and Corollary B gives a complete characterization of isolated homogeneous hypersurface singularities. Let $(V, 0)$ and $(W, 0)$ be two isolated hypersurface singularities in $\mathbb{C}^{n}$. We say that $(V, 0)$ and $(W, 0)$ have the same topological type if $\left(\mathbb{C}^{n}, V, 0\right)$ is homeomorphically equivalent to $\left(\mathbb{C}^{n}, W, 0\right)$. The famous Zariski multiplicity question asks whether $(V, 0)$ and $(W, 0)$ have the same multiplicity if they have the same topological type, i.e., whether there is only one multiplicity within the same topological type. For two-dimensional isolated quasihomogeneous singularities, the Zariski multiplicity question was solved [Yau 1988; $\mathrm{Xu}$ and Yau 1989]. Proposition 3.1 says that there are at most $\mu^{1 / n}+1$ multiplicities within the same topological type. On the other hand, Theorem C below confirms that the 1995 Yau conjecture is true for the case of $p_{g}=0$.

Theorem C. Let $f:\left(\mathbb{C}^{n}, 0\right) \rightarrow(\mathbb{C}, 0)$ be a weighted homogeneous polynomial with an isolated singularity at the origin. Let $\mu, p_{g}$ and $v$ be the Milnor number, geometric genus and multiplicity of the singularity $V=\{z: f(z)=0\}$. If $p_{g}=0$, then

$$
\mu-p(v) \geq n ! p_{g},
$$

where $p(v)=(v-1)^{n}-v(v-1) \cdots(v-n+1)(=0)$, and equality holds if and only if $f$ is a homogeneous polynomial after a biholomorphic change of coordinates.

In Section 2, we recall the material which is necessary to prove the main theorems. In Section 3, we prove the main theorems. 


\section{Preliminaries}

In this section, we recall some known results which are needed to prove the main theorems. Let $f\left(z_{1}, \ldots, z_{n}\right)$ be a germ of an analytic function at the origin such that $f(0)=0$. Suppose $f$ has an isolated critical point at the origin, and suppose $f$ can be developed in a convergent Taylor series $f\left(z_{1}, \ldots, z_{n}\right)=\sum a_{\lambda} z^{\lambda}$, where $z^{\lambda}=z_{1}^{\lambda_{1}} \cdots z_{n}^{\lambda_{n}}$. Recall that the Newton boundary $\Gamma(f)$ is the union of compact faces of $\Gamma_{+}(f)$, where $\Gamma_{+}(f)$ is the convex hull of the union of subsets $\left\{\lambda+\mathbb{R}_{+}^{n}\right\}$ for $\lambda$ such that $a_{\lambda} \neq 0$. Let $\Gamma_{-}(f)$, the Newton polyhedron of $f$, be the cone over $\Gamma(f)$ with cone point at 0 . For any closed face $\Delta$ of $\Gamma(f)$, we associate the polynomial $f_{\Delta}(z)=\sum_{\lambda \in \Delta} a_{\lambda} z^{\lambda}$. We say that $f$ is nondegenerate if $f_{\Delta}$ has no critical point in $\left(\mathbb{C}^{*}\right)^{n}$ for any $\Delta \in \Gamma(f)$, where $\mathbb{C}^{*}=\mathbb{C}-\{0\}$. We say that a point $p$ of the integral lattice $\mathbb{Z}^{n}$ in $\mathbb{R}^{n}$ is positive if all coordinates of $p$ are positive.

Theorem 2.1 [Merle and Teissier 1980]. Let $(V, 0)$ be an isolated hypersurface singularity defined by a nondegenerate holomorphic function $f:\left(\mathbb{C}^{n}, 0\right) \rightarrow(\mathbb{C}, 0)$. Then the geometric genus $p_{g}=\#\left\{p \in \mathbb{Z}^{n} \cap \Gamma_{-}(f): p\right.$ is positive $\}$.

Recall that a polynomial $f\left(z_{1}, \ldots, z_{n}\right)$ is a weighted homogeneous polynomial of type $\left(w_{1}, \ldots, w_{n}\right)$, where $w_{1}, \ldots, w_{n}$ are fixed positive rational numbers, if it can be expressed as a linear combination of monomials $z_{1}^{i_{1}} \cdots z_{n}^{i_{n}}$ for which $i_{1} w_{1}+\cdots+$ $i_{n} w_{n}=1$. As a consequence of Theorem 2.1, for an isolated singularity defined by a weighted homogeneous polynomial, computing the geometric genus is equivalent to counting the number of positive integral points in the tetrahedron defined by $x_{1} w_{1}+\cdots+x_{n} w_{n} \leq 1, x_{1} \geq 0, \ldots, x_{n} \geq 0$. We also need the following result:

Theorem 2.2 [Milnor and Orlik 1970]. Let $f\left(z_{1}, \ldots, z_{n}\right)$ be a weighted homogeneous polynomial of type $\left(w_{1}, \ldots, w_{n}\right)$ with an isolated singularity at the origin. Then the Milnor number $\mu$ is equal to $\left(1 / w_{1}-1\right) \cdots\left(1 / w_{n}-1\right)$.

Yau [1977] gave a lower bound for $p_{g}$ of a hypersurface singularity.

Theorem 2.3 [Yau 1977]. Let

$$
f\left(z_{1}, \ldots, z_{n-1}, z_{n}\right)=z_{n}^{m}+a_{1}\left(z_{1}, \ldots, z_{n-1}\right) z_{n}^{m-1}+\cdots+a_{m}\left(z_{1}, \ldots, z_{n-1}\right)
$$

be holomorphic near $(0, \ldots, 0)$. Let $d_{i}$ be the order of the zero of $a_{i}\left(z_{1}, \ldots, z_{n-1}\right)$ at $(0, \ldots, 0)$, with $d_{i} \geq i$. Let $d=\min _{1 \leq i \leq m}\left(d_{i} / i\right)$. Suppose that

$$
V=\left\{\left(z_{1}, \ldots, z_{n}\right): f\left(z_{1}, \ldots, z_{n}\right)=0\right\},
$$

defined in a suitably small polydisc, has $p=(0, \ldots, 0)$ as its only singularity. Let $\pi: M \rightarrow V$ be a resolution of $V$. Then $\operatorname{dim} H^{n-2}(M, \mathcal{O})>(m-1) d-(n-1)$.

Remark. Here, the singularity is $(n-1)$-dimensional, so $\operatorname{dim} H^{n-2}(M, \mathcal{O})=p_{g}$. 
Let $f \in \mathbb{C}\left\{z_{1}, \ldots, z_{n}\right\}$ define an isolated singularity at the origin. Let $w=$ $\left(w_{1}, \ldots, w_{n}\right)$ be a weight on the coordinates $\left(z_{1}, \ldots, z_{n}\right)$ for positive integers $w_{i}$, $i=1, \ldots, n$. We have the weighted Taylor expansion $f=f_{\rho}+f_{\rho+1}+\cdots$ with respect to $w$, where $f_{\rho} \neq 0$ and $f_{k}$ is a weighted homogeneous polynomial of type $\left(w_{1}, \ldots, w_{n} ; k\right)$ for $k \geq \rho$, i.e., $f_{k}$ is a linear combination of monomials $z_{1}^{i_{1}} \cdots z_{n}^{i_{n}}$ for which $i_{1} w_{1}+\cdots+i_{n} w_{n}=k$.

Theorem 2.4 [Furuya and Tomari 2004]. Let $f \in \mathbb{C}\left\{z_{1}, \ldots, z_{n}\right\}$ define an isolated singularity at the origin. With the above situation, then:

(1) The following inequality holds:

$$
\mu(f) \geq\left(\frac{\rho}{w_{1}}-1\right) \cdots\left(\frac{\rho}{w_{n}}-1\right) .
$$

(2) Equality holds in (1) if and only if $f_{\rho}$ defines an isolated singularity at the origin.

Here we recall that $f$ is called a semiquasihomogeneous function if the initial term $f_{\rho}$ defines an isolated singularity at the origin.

Definition 2.1. Let $f, g:\left(\mathbb{C}^{n}, 0\right) \rightarrow(\mathbb{C}, 0)$ be germs of holomorphic functions defining isolated hypersurface singularities $V_{f}=\{z: f(z)=0\}$ and $V_{g}=\{z: g(z)=0\}$. Let $\phi:\left(\mathbb{C}^{n}, 0\right) \rightarrow\left(\mathbb{C}^{n}, 0\right)$ be the germ of a biholomorphic map.

(1) $f$ is contact-equivalent to $g$ if $\phi\left(V_{f}\right)=V_{g}$.

(2) $f$ is right-equivalent to $g$ if $g=f \circ \phi$.

The Milnor number is an invariant under right-equivalence and the Tjurina number is an invariant under contact equivalence. It is a nontrivial theorem that the Milnor number is indeed an invariant under contact equivalence:

Theorem 2.5 [Greuel 1975]. Let $\mathbb{R}$ be an algebraically closed field of characteristic zero and $f, g \in \mathbb{K}\left\{z_{1}, z_{2}, \ldots, z_{n}\right\}$. If $f$ is contact-equivalent to $g$, then $\mu(f)=\mu(g)$.

Theorem 2.6 [Shoshitaishvili 1976; Benson and Yau 1990]. If $f$ and $g$ are germs of isolated weighted homogeneous singularities at the origin in $\mathbb{C}^{n}$, then $f$ and $g$ are right-equivalent if and only if $f$ and $g$ are contact-equivalent.

Theorem 2.7 [Saito 1971]. Let $f:\left(\mathbb{C}^{n}, 0\right) \rightarrow(\mathbb{C}, 0)$ be the germ of a complex analytic function with an isolated critical point at the origin.

(a) $f$ is right-equivalent to a weighted homogeneous polynomial if and only if $\mu=\tau$ or

$$
f \in J_{f}:=\left(\frac{\partial f}{\partial z_{1}}, \ldots, \frac{\partial f}{\partial z_{n}}\right)
$$


(b) If $f$ is weighted homogeneous with normalized weight system $\left(w_{1}, \ldots, w_{n}, 1\right)$ with $0<w_{1} \leq \cdots \leq w_{n}<1$ and if $f \in \boldsymbol{m}_{\mathbb{C}^{n}, 0}^{3}$, then the weight system is unique and $0<w_{1} \leq \cdots \leq w_{n}<\frac{1}{2}$.

(c) If $f \in J_{f}$, then $f$ is right-equivalent to a weighted homogeneous polynomial $g\left(z_{1}, \ldots, z_{k}\right)+z_{k+1}^{2}+\cdots+z_{n}^{2}$ with $g \in \boldsymbol{m}_{\mathbb{C}^{n}, 0}^{3}$. Specifically, its normalized weight system satisfies $0<w_{1} \leq \cdots \leq w_{k}<w_{k+1}=\cdots=w_{n}=\frac{1}{2}$.

(d) If $f$ and $\bar{f} \in \mathbb{O}_{\mathbb{C}^{n}, 0}$ are right-equivalent and weighted homogeneous with normalized weight systems $\left(w_{1}, \ldots, w_{n}, 1\right)$ and $\left(\bar{w}_{1}, \ldots, \bar{w}_{n}, 1\right)$ with $w_{1} \leq \cdots \leq w_{n} \leq \frac{1}{2}$ and $\bar{w}_{1} \leq \cdots \leq \bar{w}_{n} \leq \frac{1}{2}$, then $w_{i}=\bar{w}_{i}$.

\section{Proof of the main theorems}

The following statement is well known.

Proposition 3.1 [Teissier 1973]. Let $f:\left(\mathbb{C}^{n}, 0\right) \rightarrow(\mathbb{C}, 0)$ be a holomorphic germ defining an isolated hypersurface singularity $V=\{z: f(z)=0\}$ at the origin. Let $\mu$ and $v$ be the Milnor number and multiplicity of $(V, 0)$ respectively. Then

$$
\mu \geq(v-1)^{n},
$$

and equality holds in (3-1) if and only if $f$ is a semihomogeneous function (i.e., $f=f_{v}+g$, where $f_{v}$ is a nondegenerate homogeneous polynomial of degree $v$ and $g$ consists of terms of degree at least $v+1)$ after a biholomorphic change of coordinates.

Proof. Let $f\left(z_{1}, \ldots, z_{n}\right):\left(\mathbb{C}^{n}, 0\right) \rightarrow(\mathbb{C}, 0)$ be a holomorphic function with an isolated singularity at the origin. Let $\mu$ and $v$ be the Milnor number and multiplicity of the singularity $V=\{z: f(z)=0\}$. By an analytic change of coordinates, one can assume that the $z_{n}$-axis is not contained in the tangent cones of $V$, so that $f\left(0, \ldots, 0, z_{n}\right) \neq 0$. By the Weierstrass preparation theorem, near 0 , the germ $f$ can be represented as a product $f\left(z_{1}, \ldots, z_{n}\right)=u\left(z_{1}, \ldots, z_{n}\right) g\left(z_{1}, \ldots, z_{n}\right)$, where $u(0, \ldots, 0) \neq 0$ and

$$
g\left(z_{1}, \ldots, z_{n-1}, z_{n}\right)=z_{n}^{\nu}+a_{1}\left(z_{1}, \ldots z_{n-1}\right) z_{n}^{\nu-1}+\cdots+a_{\nu}\left(z_{1}, \ldots, z_{n-1}\right),
$$

where $v$ is the multiplicity of $f\left(z_{1}, \ldots, z_{n}\right)$ and $a_{i} \in\left(z_{1}, \ldots, z_{n-1}\right)^{i}$ for $i=1, \ldots, v$. Therefore $f\left(z_{1}, \ldots, z_{n}\right)$ is contact-equivalent to $g\left(z_{1}, \ldots, z_{n}\right)$.

Let $d_{i}$ be the order of the zero of $a_{i}\left(z_{1}, \ldots, z_{n-1}\right)$ at $(0, \ldots, 0)$, with $d_{i} \geq i$. Let $d=\min _{1 \leq i \leq v}\left(d_{i} / i\right)$; then $d \geq 1$. We define a new weight $w$ on the coordinate system: $w\left(z_{n}\right)=d, w\left(z_{i}\right)=1$ for $1 \leq i \leq n-1$. With respect to the new weights, $z_{n}^{v}$ has degree $d v$ and $a_{i}\left(z_{1}, \ldots, z_{n-1}\right) z_{n}^{v-i}$ has degree at least $d(v-i)+d_{i} \geq$ $d \nu-d i+d i=d \nu$. Thus the initial term of $f\left(z_{1}, \ldots, z_{n}\right)$ has degree $\rho=d v$. By 
Theorem 2.5, the Milnor number is an invariant under the contact equivalence. By Theorem 2.4(1), we have

$$
\mu=\mu(g) \geq\left(\frac{d v}{d}-1\right)\left(\frac{d v}{1}-1\right) \cdots\left(\frac{d v}{1}-1\right)=(v-1)(d v-1)^{n-1} \geq(v-1)^{n} .
$$

Thus, we proved the inequality (3-1).

We need to show that equality in (3-1) holds if and only if $f$ is a semihomogeneous function after a biholomorphic change of coordinates.

$\Rightarrow$ : If $\mu=(v-1)^{n}$, then by the fact that $\mu \geq(v-1)(d v-1)^{n-1} \geq(v-1)^{n}$, we have $d=1$, and by Theorem 2.4(2), $g_{d v}\left(z_{1}, \ldots, z_{n}\right)=g_{v}\left(z_{1}, \ldots, z_{n}\right)$ is a homogeneous polynomial of degree $v$ defining an isolated singularity. Hence $f\left(z_{1}, \ldots, z_{n}\right)$ is contact-equivalent to a semihomogeneous singularity $g$; i.e., $f$ is a semihomogeneous function after a biholomorphic change of coordinates.

$\Leftarrow$ : Suppose $f$ is a semihomogeneous polynomial after a biholomorphic change of coordinates. Since the Milnor number of $f$ is the same as the Milnor number of its initial part (see [Arnold 1974]) which is a homogeneous polynomial with degree $v$, so $\mu=(v-1)^{n}$ is obvious.

Proof of Theorem A. By Proposition 3.1, it is sufficient to show that if $f$ is a weighted homogeneous singularity, then $\mu=(v-1)^{n}$ if and only if $f$ is equivalent to a homogeneous singularity.

The "if" part is trivial. We only need to consider the "only if" part. By Saito's theorem (see Theorem 2.7(c)), we can choose normalized weights for $f$, which means that these weights satisfy $0<w_{i} \leq \frac{1}{2}, 1 \leq i \leq n$. By what we have proved above, we know that there exists a $g\left(z_{1}, \ldots, z_{n}\right)$ so that $f$ is contact equivalent to $g$; moreover the initial part of $g$ is $g_{v}$, a homogeneous polynomial with degree $v$, and $g_{v}$ also defines an isolated singularity at the origin. We can rewrite $f$ and $g$ as

$$
\begin{aligned}
& f\left(z_{1}, \ldots, z_{n}\right)=f_{v}\left(z_{1}, \ldots, z_{n}\right)+f_{v+1}\left(z_{1}, \ldots, z_{n}\right)+\cdots, \\
& g\left(z_{1}, \ldots, z_{n}\right)=g_{v}\left(z_{1}, \ldots, z_{n}\right)+g_{v+1}\left(z_{1}, \ldots, z_{n}\right)+\cdots,
\end{aligned}
$$

where $f_{i}$ and $g_{i}, i \geq v$, are the homogeneous parts of $f$ and $g$ respectively and $g_{v}$ defines an isolated singularity at the origin. Since for weighted homogeneous singularities contact equivalence is the same as right equivalence (see Theorem 2.6), there exists a biholomorphism at the origin:

$$
\begin{aligned}
\phi:\left(\mathbb{C}^{n}, 0\right) & \rightarrow\left(\mathbb{C}^{n}, 0\right), \\
\left(z_{1}, \ldots, z_{n}\right) & \mapsto\left(\phi_{1}\left(z_{1}, \ldots, z_{n}\right), \ldots, \phi_{n}\left(z_{1}, \ldots, z_{n}\right)\right),
\end{aligned}
$$


such that $f\left(z_{1}, \ldots, z_{n}\right)=g\left(\phi_{1}\left(z_{1}, \ldots, z_{n}\right), \ldots, \phi_{n}\left(z_{1}, \ldots, z_{n}\right)\right)$ and

$$
\begin{gathered}
\phi_{1}\left(z_{1}, \ldots, z_{n}\right)=a_{11} z_{1}+\cdots+a_{1 n} z_{n}+H_{1}^{2}+H_{1}^{3}+\cdots, \\
\vdots \\
\phi_{n}\left(z_{1}, \ldots, z_{n}\right)=a_{n 1} z_{1}+\cdots+a_{n n} z_{n}+H_{n}^{2}+H_{n}^{3}+\cdots,
\end{gathered}
$$

where $H_{i}^{j}=\sum_{\alpha_{1}+\cdots+\alpha_{n}=j} c_{i}\left(\alpha_{1}, \ldots, \alpha_{n}\right) z_{1}^{\alpha_{1}} \cdots z_{n}^{\alpha_{n}}$. Since $\phi$ is a biholomorphism at the origin, we have $\left|\left(a_{i j}\right)\right|=\operatorname{det}\left(a_{i j}\right) \neq 0$. It follows from the equality $f\left(z_{1}, \ldots, z_{n}\right)=g\left(\phi_{1}\left(z_{1}, \ldots, z_{n}\right), \ldots, \phi_{n}\left(z_{1}, \ldots, z_{n}\right)\right)$ that

$$
\begin{aligned}
g_{v}\left(\phi_{1}\left(z_{1}, \ldots, z_{n}\right), \ldots, \phi_{n}\left(z_{1}, \ldots, z_{n}\right)\right) & \\
+ & g_{v+1}\left(\phi_{1}\left(z_{1}, \ldots, z_{n}\right)\right. \\
& \left., \ldots, \phi_{n}\left(z_{1}, \ldots, z_{n}\right)\right)+\cdots \\
& =f_{v}\left(z_{1}, \ldots, z_{n}\right)+f_{v+1}\left(z_{1}, \ldots, z_{n}\right)+\cdots .
\end{aligned}
$$

Comparing the degree of each side, we have

$$
g_{v}\left(\bar{\phi}_{1}\left(z_{1}, \ldots, z_{n}\right), \ldots, \bar{\phi}_{n}\left(z_{1}, \ldots, z_{n}\right)\right)=f_{v}\left(z_{1}, \ldots, z_{n}\right),
$$

where $\bar{\phi}_{i}=a_{i 1} z_{1}+\cdots+a_{i n} z_{n}, 1 \leq i \leq n$. Since $\operatorname{det}\left(a_{i j}\right) \neq 0, f_{v}$ is right-equivalent to $g_{v}$. Therefore $f_{v}$ also defines an isolated singularity. Now we have two normalized weights for $f_{v}$ : one is $\left(w_{1}, \ldots, w_{n}\right)$, because each monomial in $f_{v}$ comes from $f$, and the other is $(1 / v, \ldots, 1 / v)$ which follows from the fact that $f_{v}$ is a homogeneous polynomial with degree $v$. By Theorem 2.7, we have $w_{1}=w_{2}=\cdots=w_{n}=1 / \nu$. Therefore $f\left(z_{1}, \ldots, z_{n}\right)=f_{v}\left(z_{1}, \ldots, z_{n}\right)$ is a homogeneous polynomial.

Proof of Corollary B. This follows from Theorem A and Theorem 2.7(a).

Proof of Theorem C. Since $p_{g}=0$, by Theorem 2.3, we have $0>(v-1) d-(n-1)$, where $d=\min _{1 \leq i \leq v}\left(d_{i} / i\right)$, and $d_{i}$ is the order of the zero of $a_{i}\left(x_{1}, \ldots, x_{n}\right)$ at $(0, \ldots, 0)$, with $d_{i} \geq i$. Then $v<(n-1) / d+1$. Since $d \geq 1, v$ is an integer at least 2 for an isolated hypersurface singularity, so we have $2 \leq v \leq n-1$. Therefore $p(v)=(v-1)^{n}-v(v-1) \cdots(v-n+1)=(v-1)^{n}$. The theorem is reduced to proving that

$$
\mu \geq(v-1)^{n},
$$

where equality holds if and only if $f$ is a homogeneous polynomial after a biholomorphic change of coordinates. The proof follows from Theorem A immediately.

\section{References}

[Arnold 1974] V. I. Arnold, "Нормальные формы функций в окрестности вырожденных критических точек", Uspekhi Mat. Nauk 29:2(176) (1974), 11-49. Translated as "Normal forms of functions in the neighborhood of degenerate critical points" in Russian Math. Surveys 29:2 (1974), 10-50. MR 58 \#24324 Zbl 0298.57022 
[Benson and Yau 1990] M. Benson and S. S.-T. Yau, "Equivalences between isolated hypersurface singularities", Math. Ann. 287:1 (1990), 107-134. MR 91h:58006 Zbl 0673.32016

[Chen et al. 2011] I. Chen, K.-P. Lin, S. S.-T. Yau, and H.-Q. Zuo, "Coordinate-free characterization of homogeneous polynomials with isolated singularities", Comm. Anal. Geom. 19:4 (2011), 661-704. MR 2880212 Zbl 1246.32030

[Furuya and Tomari 2004] M. Furuya and M. Tomari, "A characterization of semi-quasihomogeneous functions in terms of the Milnor number", Proc. Amer. Math. Soc. 132:7 (2004), 1885-1890. MR 2005f:14007 Zbl 1052.32023

[Greuel 1975] G.-M. Greuel, "Der Gauss-Manin-Zusammenhang isolierter Singularitäten von vollständigen Durchschnitten”, Math. Ann. 214 (1975), 235-266. MR 53 \#417 Zbl 0285.14002

[Lin and Yau 2004] K.-P. Lin and S. S.-T. Yau, "Classification of affine varieties being cones over nonsingular projective varieties: hypersurface case", Comm. Anal. Geom. 12:5 (2004), 1201-1219. MR 2006a:14102 Zbl 1072.32022

[Lin et al. 2006a] K.-P. Lin, Z.-H. Tu, and S. S.-T. Yau, "Characterization of isolated homogeneous hypersurface singularities in $\mathbb{C}^{4,}$, Sci. China Ser. A 49:11 (2006), 1576-1592. MR 2008c:32037 Zbl 1115.32017

[Lin et al. 2006b] K.-P. Lin, X. Wu, S. S.-T. Yau, and H.-S. Luk, "A remark on lower bound of Milnor number and characterization of homogeneous hypersurface singularities", Comm. Anal. Geom. 14:4 (2006), 625-632. MR 2008a:32026 Zbl 1111.32025

[Merle and Teissier 1980] M. Merle and B. Teissier, "Conditions d'adjonction: d'après Du Val", pp. 229-245 in Seminaire sur les singularités des surfaces (Palaiseau, 1976-1977), edited by M. Demazure et al., Lecture Notes in Math. 777, Springer, Berlin, 1980. Zbl 0461.14009

[Milnor and Orlik 1970] J. Milnor and P. Orlik, "Isolated singularities defined by weighted homogeneous polynomials", Topology 9 (1970), 385-393. MR 45 \#2757 Zbl 0204.56503

[Saito 1971] K. Saito, “Quasihomogene isolierte Singularitäten von Hyperflächen”, Invent. Math. 14 (1971), 123-142. MR 45 \#3767 Zbl 0224.32011

[Shoshitaishvili 1976] A. N. Shoshitaishvili, "О функциях с изоморфными Якобиевыми идеалами", Funkcional. Anal. i Priložen. 10:2 (1976), 57-62. Translated as "Functions with isomorphic Jacobian ideals" in Funct. Anal. Appl. 10:2 (1976), 128-133. MR 54 \#5492 Zbl 0346.32023

[Teissier 1973] B. Teissier, "Cycles évanescents, sections planes et conditions de Whitney", pp. 285-362 in Singularités à Cargèse (Cargèse, 1972), Astérisque 7-8, Société Mathématique de France, Paris, 1973. MR 51 \#10682 Zbl 0295.14003

[Xu and Yau 1989] Y.-J. Xu and S. S.-T. Yau, "Classification of topological types of isolated quasi-homogeneous two-dimensional hypersurface singularities", Manuscripta Math. 64:4 (1989), 445-469. MR 91e:32034 Zbl 0681.32008

[Xu and Yau 1992] Y.-J. Xu and S. S.-T. Yau, "A sharp estimate of the number of integral points in a tetrahedron”, J. Reine Angew. Math. 423 (1992), 199-219. MR 93d:11067 Zbl 0734.11048

[Xu and Yau 1993] Y.-J. Xu and S. S.-T. Yau, "Durfee conjecture and coordinate free characterization of homogeneous singularities", J. Differential Geom. 37:2 (1993), 375-396. MR 94f:32073 Zbl 0793.32016

[Xu and Yau 1996] Y.-J. Xu and S. S.-T. Yau, "A sharp estimate of the number of integral points in a 4-dimensional tetrahedra", J. Reine Angew. Math. 473 (1996), 1-23. MR 97d:11151 Zbl 0844.11063

[Yau 1977] S. S.-T. Yau, "Two theorems on higher dimensional singularities", Math. Ann. 231:1 (1977), 55-59. MR 58 \#11511 Zbl 0343.32010

[Yau 1988] S. S.-T. Yau, "Topological types and multiplicities of isolated quasihomogeneous surface singularities”, Bull. Amer. Math. Soc. (N.S.) 19:2 (1988), 447-454. MR 92b:32042 Zbl 0659.32013 
[Yau and Zuo 2012] S. S.-T. Yau and H.-Q. Zuo, "Lower estimate of Milnor number and characterization of isolated homogeneous hypersurface singularities", Pacific J. Math. 260:1 (2012), 245-255. MR 3001794 Zbl 1276.32022

Received February 8, 2014.

STEPHEN YAU

DEPARTMENT OF MATHEMATICAL SCIENCES

TSINGHUA UNIVERSITY

BEIJING, 100084

CHINA

yau@uic.edu

HuAiqing ZuO

Mathematical Sciences Center

TSINGHUA UNIVERSITY

BEIJING, 100084

CHINA

hqzuo@math.tsinghua.edu.cn 


\title{
PACIFIC JOURNAL OF MATHEMATICS
}

\author{
msp.org/pjm
}

Founded in 1951 by E. F. Beckenbach (1906-1982) and F. Wolf (1904-1989)

\section{EDITORS}

Don Blasius (Managing Editor)

Department of Mathematics

University of California

Los Angeles, CA 90095-1555

blasius@math.ucla.edu

\author{
Paul Balmer \\ Department of Mathematics \\ University of California \\ Los Angeles, CA 90095-1555 \\ balmer@math.ucla.edu \\ Robert Finn \\ Department of Mathematics \\ Stanford University \\ Stanford, CA 94305-2125 \\ finn@math.stanford.edu \\ Sorin Popa \\ Department of Mathematics \\ University of California \\ Los Angeles, CA 90095-1555 \\ popa@math.ucla.edu
}

\author{
Vyjayanthi Chari \\ Department of Mathematics \\ University of California \\ Riverside, CA 92521-0135 \\ chari@math.ucr.edu \\ Kefeng Liu \\ Department of Mathematics \\ University of California \\ Los Angeles, CA 90095-1555 \\ liu@math.ucla.edu \\ Jie Qing \\ Department of Mathematics \\ University of California \\ Santa Cruz, CA 95064 \\ qing@ cats.ucsc.edu
}

\section{PRODUCTION}

Silvio Levy, Scientific Editor, production@msp.org

\section{SUPPORTING INSTITUTIONS}

ACADEMIA SINICA, TAIPEI

CALIFORNIA INST. OF TECHNOLOGY

INST. DE MATEMÁTICA PURA E APLICADA

KEIO UNIVERSITY

MATH. SCIENCES RESEARCH INSTITUTE

NEW MEXICO STATE UNIV.

OREGON STATE UNIV.

\author{
STANFORD UNIVERSITY \\ UNIV. OF BRITISH COLUMBIA \\ UNIV. OF CALIFORNIA, BERKELEY \\ UNIV. OF CALIFORNIA, DAVIS \\ UNIV. OF CALIFORNIA, LOS ANGELES \\ UNIV. OF CALIFORNIA, RIVERSIDE \\ UNIV. OF CALIFORNIA, SAN DIEGO \\ UNIV. OF CALIF., SANTA BARBARA
}

\author{
Daryl Cooper \\ Department of Mathematics \\ University of California \\ Santa Barbara, CA 93106-3080 \\ cooper@math.ucsb.edu \\ Jiang-Hua Lu \\ Department of Mathematics \\ The University of Hong Kong \\ Pokfulam Rd., Hong Kong \\ jhlu@maths.hku.hk \\ Paul Yang \\ Department of Mathematics \\ Princeton University \\ Princeton NJ 08544-1000 \\ yang@math.princeton.edu
}

These supporting institutions contribute to the cost of publication of this Journal, but they are not owners or publishers and have no responsibility for its contents or policies.

See inside back cover or msp.org/pjm for submission instructions.

The subscription price for 2015 is US \$420/year for the electronic version, and \$570/year for print and electronic.

Subscriptions, requests for back issues and changes of subscribers address should be sent to Pacific Journal of Mathematics, P.O. Box 4163, Berkeley, CA 94704-0163, U.S.A. The Pacific Journal of Mathematics is indexed by Mathematical Reviews, Zentralblatt MATH, PASCAL CNRS Index, Referativnyi Zhurnal, Current Mathematical Publications and Web of Knowledge (Science Citation Index).

The Pacific Journal of Mathematics (ISSN 0030-8730) at the University of California, c/o Department of Mathematics, 798 Evans Hall \#3840, Berkeley, CA 94720-3840, is published twelve times a year. Periodical rate postage paid at Berkeley, CA 94704, and additional mailing offices. POSTMASTER: send address changes to Pacific Journal of Mathematics, P.O. Box 4163, Berkeley, CA 94704-0163.

PJM peer review and production are managed by EditFLOW ${ }^{\circledR}$ from Mathematical Sciences Publishers.

\section{PUBLISHED BY}

\section{mathematical sciences publishers \\ nonprofit scientific publishing}

http://msp.org/

(C) 2015 Mathematical Sciences Publishers 


\section{PACIFIC JOURNAL OF MATHEMATICS}

Volume $273 \quad$ No. $1 \quad$ January 2015

Maximal estimates for Schrödinger equations with inverse-square potential 1

ChangXing MiaO, JUNYong Zhang and JiQiang Zheng

Vassiliev Invariants of Virtual Legendrian Knots

PATRICIA CAHN and ASA LEVI

Some results on the generic vanishing of Koszul cohomology via

deformation theory

JIE WANG

Conformal metrics with constant curvature one and finitely many conical

singularities on compact Riemann surfaces

QING Chen, WeI WANG, Yingyi Wu and BIN XU

$\mathbb{Q}$-bases of the Néron-Severi groups of certain elliptic surfaces

MASAMICHI KURODA

On a prime zeta function of a graph

TAKehiro Hasegawa and SEIKEN SAIto

On Whittaker modules for a Lie algebra arising from the 2-dimensional torus 147

SHaObin TAN, QING WANG and ChENGKANG XU

Fréchet quantum supergroups

AXEL DE GOURSAC

Generators of the Gauss-Picard modular group in three complex dimensions

BaoHua Xie, JieYan Wang and YuePing Jiang

Complete characterization of isolated homogeneous hypersurface singularities

STEPHEN YAu and HuAiQING ZuO

A theorem of Mœglin and Waldspurger for covering groups

SHIV PRAKASH PATEL

Spanning trees and random walks on weighted graphs 